\title{
User-Based Interaction for Content-Based Image Retrieval by Mining User Navigation Patterns.
}

\author{
A.Srinagesh ${ }^{1}$ \\ CSE Department, RVR \& JC College of Engineering \\ Guntur-522019. India \\ G.P.Saradhi Varma ${ }^{2}$ \\ IT Department, SRKR College of Engineering \\ Bhimavaram-534204, India
}

\begin{abstract}
In Internet, Multimedia and Image Databases image searching is a necessity. Content-Based Image Retrieval (CBIR) is an approach for image retrieval. With User interaction included in CBIR with Relevance Feedback (RF) techniques, the results are obtained by giving more number of iterative feedbacks for large databases is not an efficient method for realtime applications. So, we propose a new approach which converges rapidly and can aptly be called as Navigation PatternBased Relevance Feedback (NPRF) with User-based interaction mode. We combined NPRF with RF techniques with three concepts viz., query Re-weighting (QR), Query Expansion (QEX) and Query Point Movement (QPM). By using, these three techniques efficient results are obtained by giving a small number of feedbacks. The efficiency of the proposed method with results is proved by calculating Precision, Recall and Evaluation measures.
\end{abstract}

Keywords-Image Retrieval; CBIR; Relevance Feedback; Navigation Patterns; Query Expansion; Query Reweighting; Query Point Movement.

\section{INTRODUCTION}

The popularity of an image retrieval system plays an important role in it's usage and application which, in turn is dependent on it's implementation. Image retrieval systems such as CBIR take a great challenge of retrieving images from a large database. Everywhere, we see the usage of images and image retrieval technique plays a vital role in different application areas like for ex: Medical Diagnosis, Military, Retail catalogs etc. There are many traditional approaches for information retrieval, but they can't satisfy the user's need to retrieve images upto a satisfactory level. CBIR techniques were firstly introduced by Rui, Hunag, and Chang, in late 1990s. There are some CBIR techniques which are search or retrieval type with browsing as a major mode of querying. CBIR is based on navigating an image collection of size 35,000 along conceptual dimensions that describes images in the collection is a very much useful method. It can also be used for intelligent image retrieval and browsing using semantic web-based techniques. All systems introduced for automatically classifying images gathered on the Web are based on the CBIR system. Another example system is art image retrieval based on user profiles is developed and it uses probabilistic support vector machines (SVM) to model user

\author{
Lavanya Thota ${ }^{1}$ \\ Guntur-522019. India. \\ A.Govardhan ${ }^{3}$ \\ CSE Department, JNTUH \\ Hyderabad-500085. India
}

CSE Department, RVR \& JC College of Engineering profiles. The same method is presented for automatic image annotation using cross media relevance models. Current interfaces of CBIR system describes an alternative interface based on a study of how home users use traditional ways of storing and organizing personal photo collections, but leveraging new possibilities enabled by digital media is not attempted. Some of researchers proposed approaches related to CBIR that involves multiple sources of information like text, HTML tags which are required to search for the images.

Several scenarios exist where medical practitioners can benefit from the use of these types of relevance feedback systems. Feedback functionality is to be provided for radiologists in assessing medical images, which is used in medical diagnosis. It is also useful as a clinical tool or in an academic context where students can benefit from access to similar diagnosed data. Content-based access to medical images has strong impacts for computer-aided diagnosis, evidence-based medicine. For each application, a certain GUI is composed and connected to the IRMA core hosting the database as well as the programs for feature extraction and comparison. However, several mechanisms are of major importance in every image retrieval system.

Feature extraction [12] is one of the ways to retrieve an image. Feature extraction plays a major role in CBIR systems. Mapping the image pixels into the feature space is feature extraction. By using this extracted feature we can search, index and browse the image from the stored database. This feature can be used to measure the similarity between the stored images.

Image retrieval approaches are based on the computing the similarity between the input query image and database images via Query by Example (QBE) system [9]. The problem occurred in this is extracted visual features are too diverse to be captured with the concept of query given by user. To, solve such problem in QBE system, user need to provide feedback like pick relevant images from retrieval of images iteratively, the feedback procedure is called Relevance Feedback (RF). This feedback is given up to user satisfaction with the retrieval results.

To solve problems we propose an approach called Navigation-Pattern-Based Relevance Feedback (NPRF) which 
is used to get efficient image retrieval quality with user interaction. CBIR combined with RF is a widely used technique to get high quality of image retrieval. Relevance feedback is a tool where end user involvement is more to improve the performance of the system. CBIR with RF methods are combined to discover Navigation Patterns i.e. user intentions taken in to account.

\section{RELATED WORK}

The term Content-Based Image Retrieval (CBIR) seems to have originated with the work of Kato for the automatic retrieval of the images from a database, based on the color and shape present. Hiremath and Pujari proposed CBIR system is used to partitioning the image into tiles by using color, texture and shape features. In earlier studies for RF make use of existing machine learning techniques to achieve semantic image retrieval which includes Statistics, EM, KNN, etc. Although these were formulating the special semantic features for image retrieval, e.g., Photobook [5], Visual SEEK [7], QBIC [1], there still have imperfect descriptions for semantic features.

Relevance feedback (RF) [2], [8], [13] is used to increase the accuracy of image search process. Relevance feedback was first proposed by Rui et. al as an interactive tool in content-based image retrieval. PFRL methods is one of the method for relevance feedback to retrieve images are used to compute local feature relevance. By giving input query image to the system then top $\mathrm{N}$ similar results are display to the end user. The user needs to give feedback like select all similar images which are relevant to the query image given by end user. Here we classify the $\mathrm{N}$ images into clusters one is containing similar images and other containing dissimilar images, the features of two clusters are averaged. To improve the result, retain all the relevant images are selected by the user and discard the irrelevant images. These discarded images are replaced by new images from the database by comparing the feature vector of the images with the mean of the similar image cluster. This process is continued up to user retrieves relevant images.

\section{A. Query-Point Movement}

For obtaining accuracy of image retrieval is moving the query-point towards the contour of the user's preference in feature space. At each feedback QPM regards multiple positive examples as a new query point. According to user's interest query point should be close to a convex region. The space-vector formula is proposed by Rocchio is as follows:

$Q_{i}=Q_{i-1}+\alpha \sum_{j=1}^{n r} \frac{R_{j}}{n r}-\beta \sum_{j=1}^{n i r} \frac{I R_{j}}{n i r}(1)$

Where, $Q_{i}$ is the vector of the $i^{\text {th }}$ query, $\mathrm{R}_{\mathrm{j}}$ is the vector of the $\mathrm{j}^{\text {th }}$ relevant image, $\mathrm{IR}_{\mathrm{j}}$ is the vector of $\mathrm{j}^{\text {th }}$ irrelevant image, $\mathrm{nr}$ is the cardinality of relevant images, nir is the cardinality of irrelevant images. There are many approaches one of them is modified version of MARS [4]. In that weighted Euclidean distance to compute the similarity between the query image and targets. The other well known study is MindReader [3], is to generalize Euclidean distance to compute targets. The modified query point of each feedback moves toward local optimal centroid.

\section{B. Query Re-weighting:}

In query reweighting if the $i^{\text {th }}$ feature $f_{i}$ exists in positive examples frequently, the system assigns a high degree to $f_{i}$ .QR like approaches proposed by the Rui et al. [6] which convert image feature vectors to weighted feature vectors in early version of Multimedia Analysis and Retrieval System (MARS). NNEW, developed by You et al. [12], the user learns query from positive and negative examples by weighting the important features. In RF approach feature weights are updated dynamically to connect low-level visual features and high-level human concepts. The search area is continuously updated by reweighting the features some targets are lost.

\section{Query Expansion:}

QPM and QR cannot elevate the quality of RF and cannot completely satisfy the user's interest spreading the feature space. QEX is the technique which solves the problem and gives the high quality of image retrieval. In this method user need to submit a query which captures an initial set of results, from these set of results number images should be relevant. $\mathrm{Wu}$ et al. [10] proposed FALCON, is designed to handle disjunctive queries within arbitrary metric spaces. Qcluster, developed by Kim and Chung [14], intends to handle the disjunctive queries by employing adaptive classification and cluster merging methods. The system expands the query based upon the terms in the selected images. This technique is used in search engines. When user has already found a set of appropriate results, then they may not desire to expand the query more.

\section{Hybrid Approach:}

Hybrid is another type of RF techniques; this method is used very little. Hybridized work focuses on the long-term usage log coming from various users. The greater effectiveness of the multisystem requires high computation cost due to multiple processing's. One of the hybrid RF techniques is IRRL proposed by Yin et al. [11]. The problem occurring in hybrid RF is that one cannot avoid the overhead of long iterations of feedback. Visual diversity existing in global feature space cannot be resolved with this technique. So, we are not using it in our proposed approach.

\section{OUR METHODOLOGY}

Our proposed approach is NPRF, which integrates the discovered patterns and RF techniques to achieve effective results.

\section{A. Outline of Navigation-Pattern Based Relevance Feedback}

To solve the problems occurred in existing approaches NPRF approach is introduced. It is solution for getting high quality of image retrieval. NPRF approach is divided into two major phases those are online image retrieval and offline knowledge discovery. Each phase contains sub phases, query image is given to the system and it finds the most similar images without considering any feature vectors then it returns a set of most relevant images. The first query process is called initial feedback. If initial feedback is satisfy by the user then system is terminated. Then positive examples are picked up by the end user and send feedback to the image search phase by including new feature weights, new query points and user's 
feedback. Then by using the navigation patterns with navigation pattern-based relevance feedback search (NPRF search) is used to find the similar images. At each feedback the results are given to the end user and related browsing information is stored in the log database.

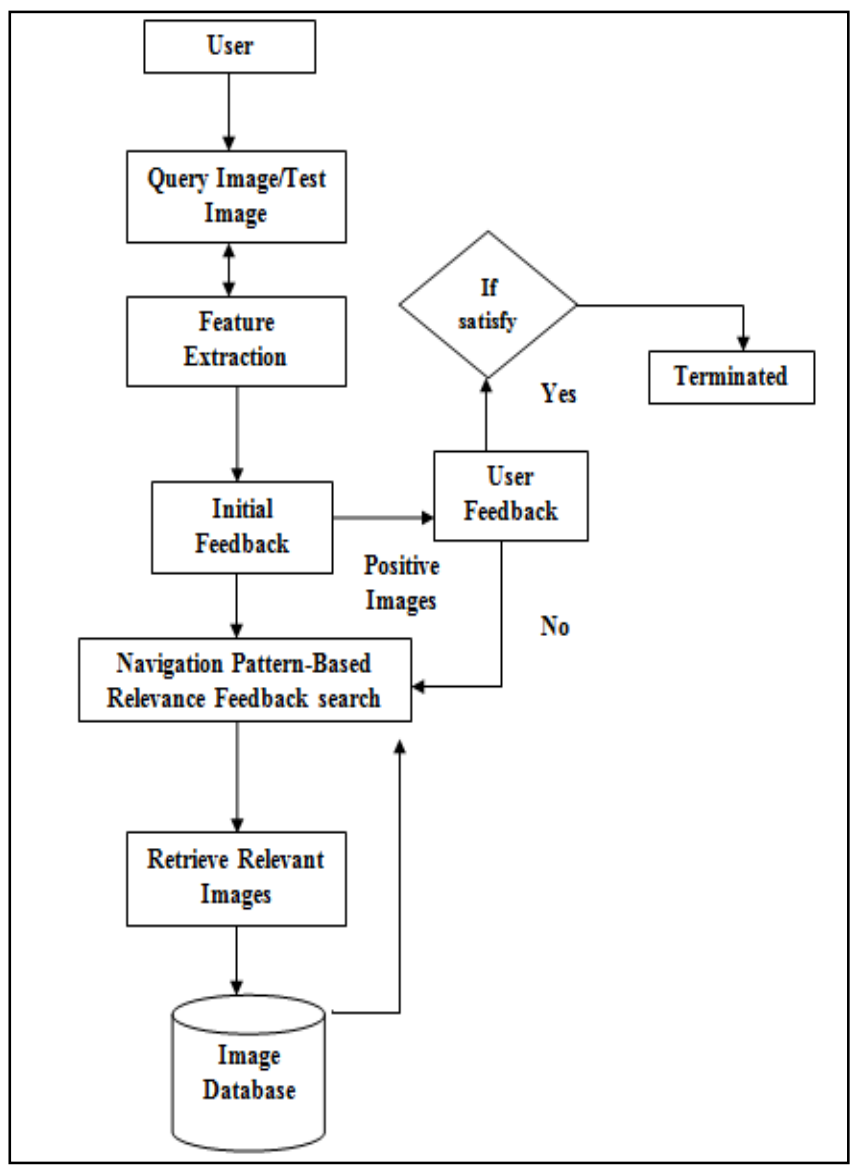

Fig. 1. Architecture of NPRF

In the above Figure, the architecture is divided in to two operations. The two steps those are Initial query processing phase and image search phase. After initial query processing phase is completed initial feedback (iteration 0) is given by this phase.

In our approach user feedback is taken into account. so, user need to give feedback as one picks positive examples, if user satisfied then system is terminated otherwise go to our proposed search NPRF search then by discovering navigation patterns relevant images are obtained.

\section{1) Image Retrieval}

In this phase we have sub phases those are Initial Query Processing Phase and Image search Phase.

\section{a) Initial Query Processing Phase:}

In this phase without considering any feature weights system extracts the visual features from original query image and similar images. The positive examples or good examples are picked up by the end user is called initial feedback or iteration0.

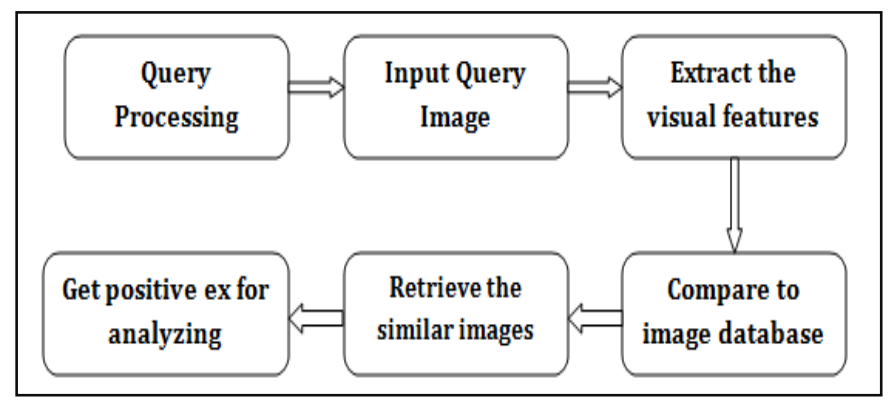

Fig. 2. Initial Query Processing Phase

\section{b) Image Search Phase:}

In this phase the intent is to extend one's search point to multiple search points by integrating the navigation patterns and the proposed search algorithm NPRF search. In this phase user intension is successfully implied. A new query point is generated at each feedback by using preceding positive examples. The search procedure is continuing up to user is satisfied.

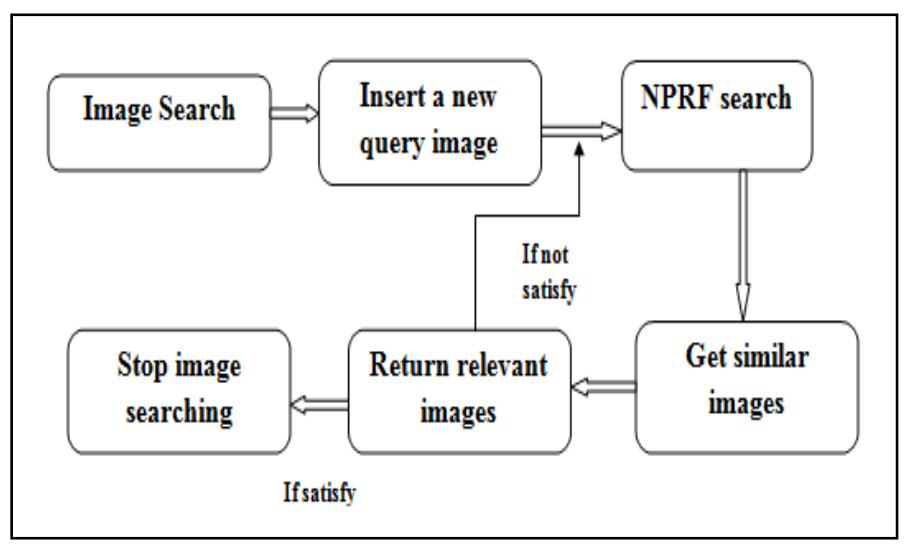

Fig. 3. Image Search Phase

\section{B. Image Search Phase}

The aim of the search strategy is to solve the problems in existing approaches; these problems result in large limitation in RF. By using RF query refinement strategies the results generated by multiple query refinement systems produce better results than individual systems. Our proposed approach NPRF Search resolves problems by using the generated navigation patterns. For the problem of existing problems like exploration convergence and redundant browsing, our proposed approach extends the search range from a query point to a number of relevant navigation paths; as a result user's interest is satisfied. The discovered navigation patterns are taken as the shortest paths to derive the efficient results in a few feedback processes. Because of high cost of navigation process for the massive image databases iterative search can be a solution. The NPRF Search algorithm can be divided as an important part of our proposed iterative solution to RF, which is combination of QEX, QP, and QR strategies.

\section{a) Flow Chart For NPRF Approach}

Flow chart of the proposed approach is shown below in fig 6. It represents a step by step procedure. Firstly a set of images 
are taken for which old query points are found and then user need to pick the positive examples to generate the new query points and these points are stored in a database, negative examples are appended to negative image set $\mathrm{N}[\mathrm{i}]$. At each feedback negative images are eliminated and no. of iterations are computed as we return and again go to step 1(feedback procedure) and system goes to step 2(Iteration process) where we get relevant or positive images without exceeding threshold thrd. Top s visual query points are generated and negative images are neglected, finally we get top $\mathrm{k}$ relevant examples.

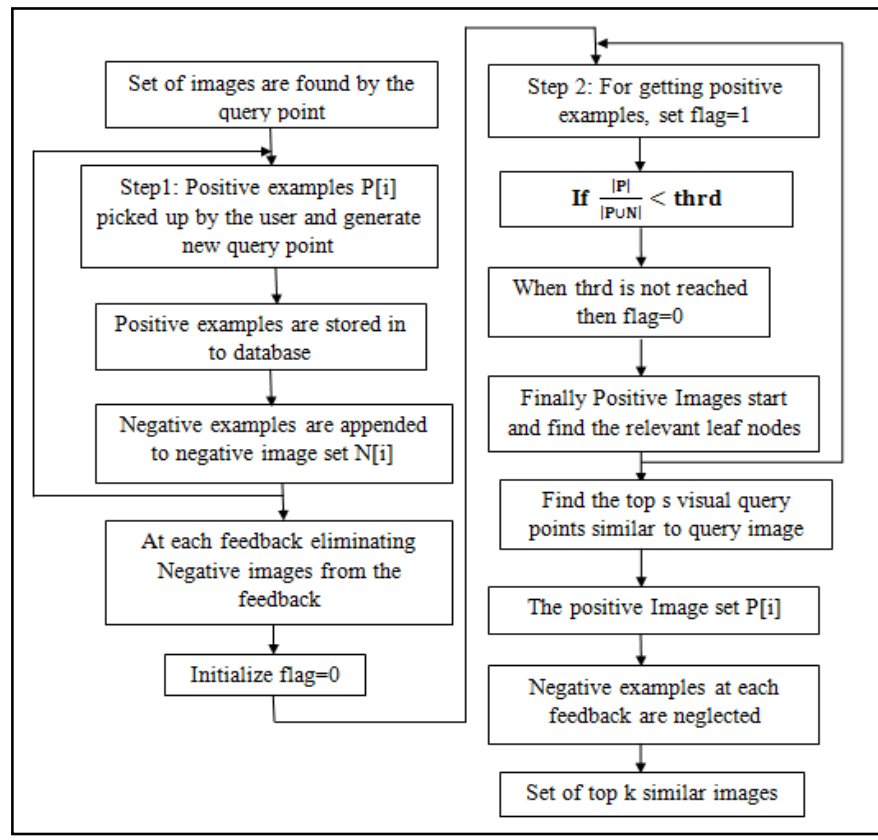

Fig. 4. Flow Chart of NPRF approach

\section{A. Algorithm NPRF Search}

NPRF Search is proposed to reach the high precision of image retrieval in a shorter query process by using the valuable navigation patterns. We explain the details of NPRF Search given below

As illustrated in NPRF Search algorithm is triggered by receiving:

a) A set of positive examples $P[i]$ and negative examples $N[i]$ determined by the user at the preceding feedback.

\section{b) A set of navigation patterns.}

In brief, the iterative search procedure can be decomposed into several steps as follows:

Figure a. A new query point is generated by averaging the visual features of positive examples.

Figure $b$. Find the similar images by determining the nearest to query image.

Figure c. Find the nearest images from the similar navigation patterns.

Figure d. Find the top s relevant visual query points from the set of the nearest images.
Figure e. Finally, the top $\mathrm{k}$ relevant images are returned to the user.

From the aspect of NPRF Search, step 1 can be done by QPM and steps 2-5 can be done by QEX. For QR, the feature weights are updated iteratively based on the positive examples at each feedback. The proposed NPRF Search takes advantage of Query refinement strategies and navigation patterns are used to make RF more efficiently and effectively. Without using navigation patterns, proposed search cannot reach the high quality of RF.

The goal of our approach is to satisfy each query efficiently instead of providing personalized functions for each user. By collecting a number of query transactions, most queries can be satisfy user's interests by NPRF Search. The details of the NPRF Search algorithm are described as follows:

\section{Algorithm of NPRF Search:}

Input: A set of positive images $\mathrm{P}$ [i] picked up by user, a set of negative set $\mathrm{N}[\mathrm{i}]$;

Output: A set of relevant images R[i];

Step 1: Generate a new query points and features $\sum_{i=1}^{n} F_{i}$ of positive images.

Step 2: let Negative images are stored in the Negative image set $\mathrm{N}$ [i]

Step 3: Initialize flag $=0$;

for each query image belongs to $\mathrm{P}[\mathrm{i}] \mathrm{do}$

Determine the images with the shortest distance

to query image $D_{i}=\min \sum_{i=1}^{n}\left(T_{i}-Q_{i}\right)$;

end for

if threshold exceeds then

for each negative image belongs to $\mathrm{N}[\mathrm{i}]$ do determine the images with the shortest distance to negative images.

\section{end for}

end if

if flag $=1$ then

Find the set of visual query points with in the retrieved images.

\section{end if}

for $\mathrm{i}=1$ to $\mathrm{k}$ do

find the relevant image set $\mathrm{R}[\mathrm{i}]$ from the database

\section{end for}

eliminate the negative images from retrieved images.

return the retrieved image set $\mathrm{R}$ [i] of top $\mathrm{k}$ similar images.

Fig. 5. Algorithm Of NPRF Search

Query point generation: This operation is used to find the images may or may not use the similarity function. Modification of the query point moves query point towards the targets in search process. Assume that a set of images is found by the query points at the previous feedback. Then visual features of the positive examples $\mathrm{P}$ [i] picked up by the user are first averaged into a new query point. From above Fig:7, consider a set of positive examples $\mathrm{P}[\mathrm{i}]$ and $d$ dimensions of the $\mathrm{i}^{\text {th }}$ feature $F_{i}=\left\{f_{1}^{x}, f_{2}^{x}, \ldots \ldots, f_{d}^{x}\right\}$ extracted 
from the $x^{\text {th }}$ positive exampleand the positive examples are stored into the database to enhance the knowledge database. The negative examples are appended to the accumulated negative set $\mathrm{N}[\mathrm{i}]$. At each feedback, eliminating negative images from the targets can increase the precision of image retrieval significantly. In addition to generating new query points and negative images, the vectors of each feature has to be calculated to keep searching the images similar to qpnew. The feature vector for similarity computation is normalized as follows:

$\sqrt{\left(X_{1}-X_{2}\right)^{2}+\left(Y_{1}-Y_{2}\right)^{2}+\left(Z_{1}-Z_{2}\right)^{2}}$

Query expansion: To solve the problem of exploration convergence, this operation is used to cover all possible results by the similar patterns discovered. QEX operation first determines query seed which is nearest to each of P[i], called positive query seed, and query seed which is nearest to each of N[i], called negative query seed. From above Fig: 7 say how to find the positive and negative query seed sets.

As a result, a set of positive query seeds is selected to start the potential search paths. Because of slight loss of the information in the negative examples is also deliberated. The desired results are more precisely by discarding negative query seeds at each feedback. At each feedback there exist some query seeds which are belonging to both positive query seed set and the negative query seed set. By dropping the negative query seeds would lead to the loss of positive query seeds i.e. these dropped negative seeds may be the start of good search paths or taken for next iteration. Both positive and negative information simultaneously taken into account. If the seed gets the maximum number of negative examples or no positive example is said as bad manner, i.e., flag=0, as shown in Fig: 7.

Otherwise, flag $=1$ for any good manner i.e. all positive examples. By considering both positive and negative information the computation cost is more. To reduce the cost of computation, at each feedback proposed algorithm assigns a bad manner to the seed that had maximum number of negative examples, if and only if the satisfaction rate $(|P| /|P \cup N|)$ cannot reach the presetting threshold thrd. Finally, the good manners are the starts of the referred navigation paths to find the relevant leaf nodes.

\section{DATASETS, EXPERIMENTAL RESULTS}

In corel database consists of seven data sets are composed of different kinds of categories. In each category contains 200 images. In our project we take a dataset consists of categories are Flowers, Animals, Mountains and Vehicles. This project is work with different databases like medical database, Wang database.

\section{A. Datasets}

For Experimentation, corel image database and web images are used in the approach. Image database which consists of corel database images, we take some of the 6 different categories from corel database those are shown in table: 1.This is also work with Wang database.
TABLE I. DATASETS

\begin{tabular}{|c|c|c|}
\hline Data Set & No. of images & \multicolumn{1}{c|}{ Category set } \\
\hline 1 & $\begin{array}{c}\text { Image database } \\
\text { (300 images })\end{array}$ & $\begin{array}{c}\text { Buses, Flowers, Mountains, } \\
\text { Horses, Elephants and Dinosaurs }\end{array}$ \\
\hline $\mathbf{2}$ & Wang database & $\begin{array}{l}\text { Buses, Flowers, Mountains, } \\
\text { Horses, Elephants and Dinosaurs }\end{array}$ \\
\hline
\end{tabular}

Experiments Datasets: By our proposed approach we have done calculations for precision, recall and evaluation measures for data set 1 .

Data Set 1:In below table: 2 we have different categories those are buses, flowers, mountains, horses, Elephants and dinosaurs which are in corel database category.

TABLE II. CALCULATIONS FOR COREL DATABASE

\begin{tabular}{|c|c|c|c|c|}
\hline Category & Precision & Recall & \multicolumn{2}{|c|}{ Evaluation Measure } \\
\cline { 3 - 5 } & & & & \multicolumn{2}{|c|}{ b=0.5 } & b=2 \\
\hline Buses & 0.416 & 0.086 & 0.8669 & 0.9562 \\
\hline Dinosaurs & 1 & 0 & 1 & 1 \\
\hline Elephants & 0.76 & 0.033 & 0.8892 & 0.9689 \\
\hline Flowers & 1 & 0 & 1 & 1 \\
\hline Horse & 0.833 & 0.083 & 0.7397 & 0.9150 \\
\hline Mountains & 0.25 & 0.25 & 0.8437 & 0.9264 \\
\hline
\end{tabular}

Average Precision Value is 0.709833 and Average Recall Value is 0.075383 .

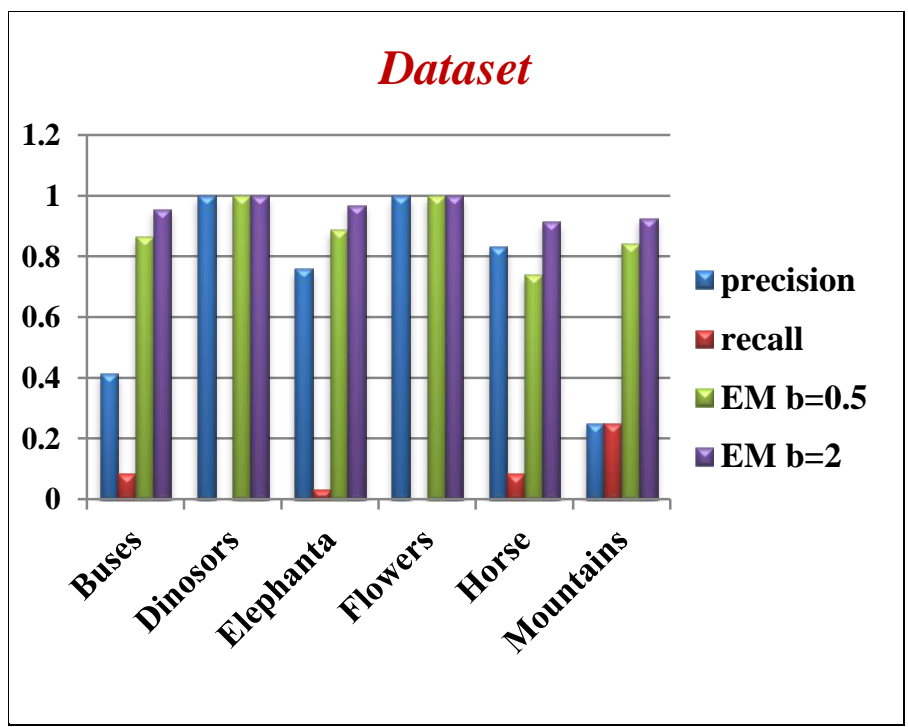

Fig. 6. Graph for Dataset Precision, recall and Evaluation measure Values

The above graph shows range of precision, recall and evaluation measure for dataset 1 , where we take evaluation measure for precision values i.e. $b=0.5$ and $b=2$. 


\section{B. Experimental Results}

Home Page:

We need to give input image and another image from image database and then click retrieve image.

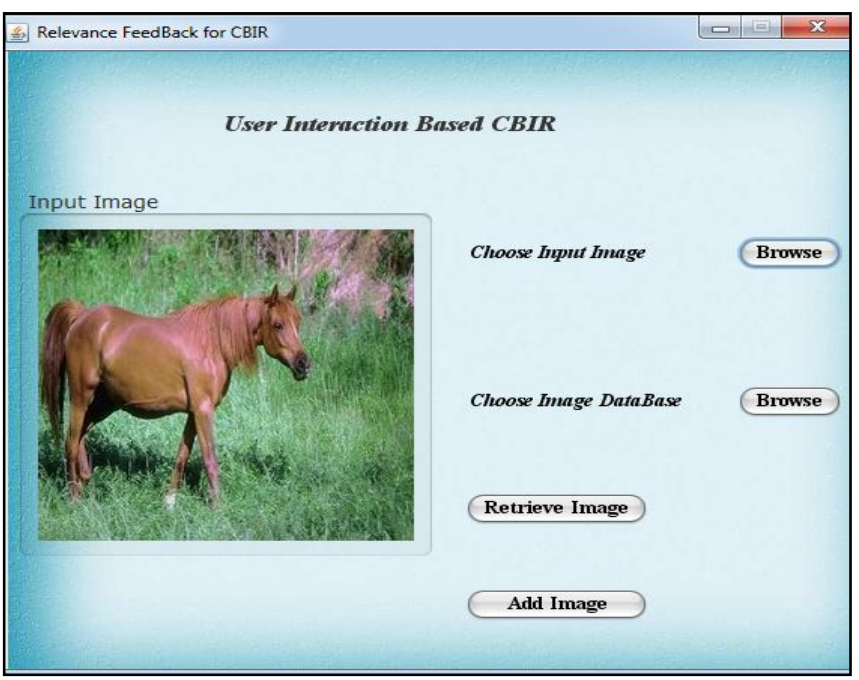

Fig. 7. Home Page

Initial Feedback:

After clicking retrieve image we get initial feedback with relevant and irrelevant images then user need to give feedback by clicking on checkbox.

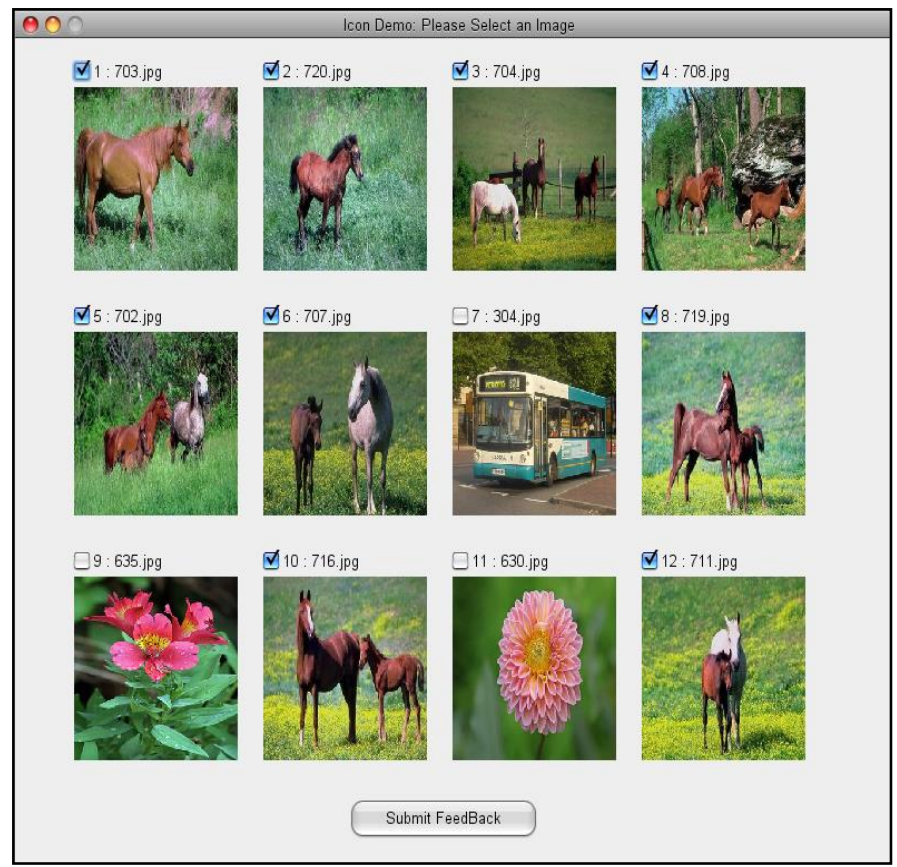

Fig. 8. Initial Feedback

\section{User feedback:}

User need to give feedback by clicking checkbox on positive image generated in initial feedback. After click submit feedback.

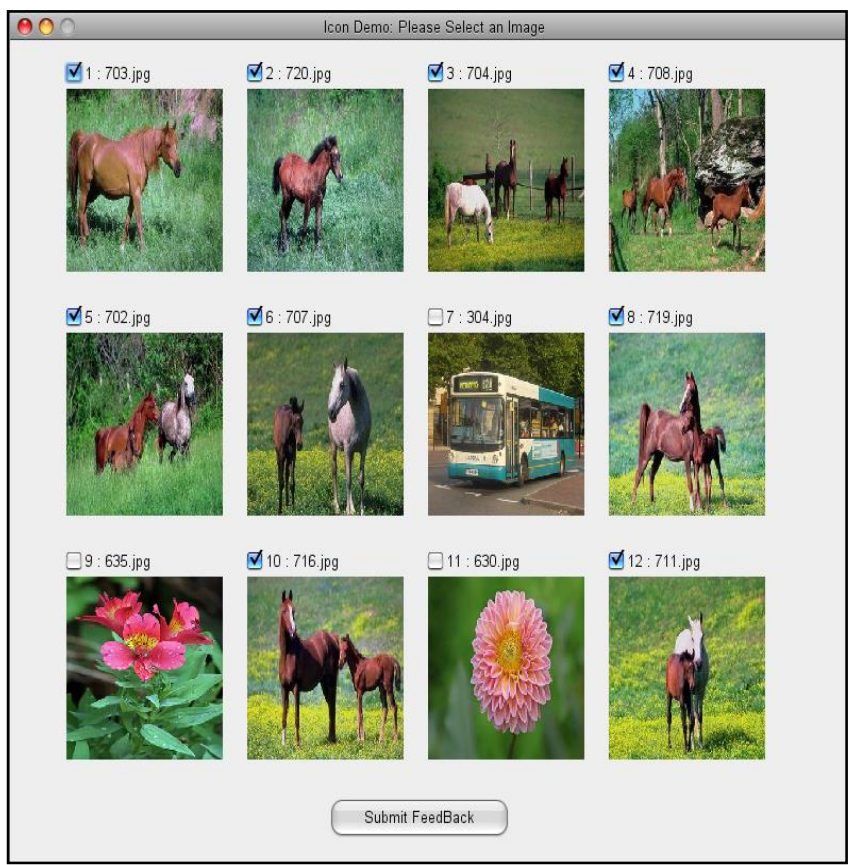

Fig. 9. User feedback

User Satisfaction:

Here user need to satisfy by result, if we click 'yes' then system is terminated otherwise we click 'no' then go to NPRF search.

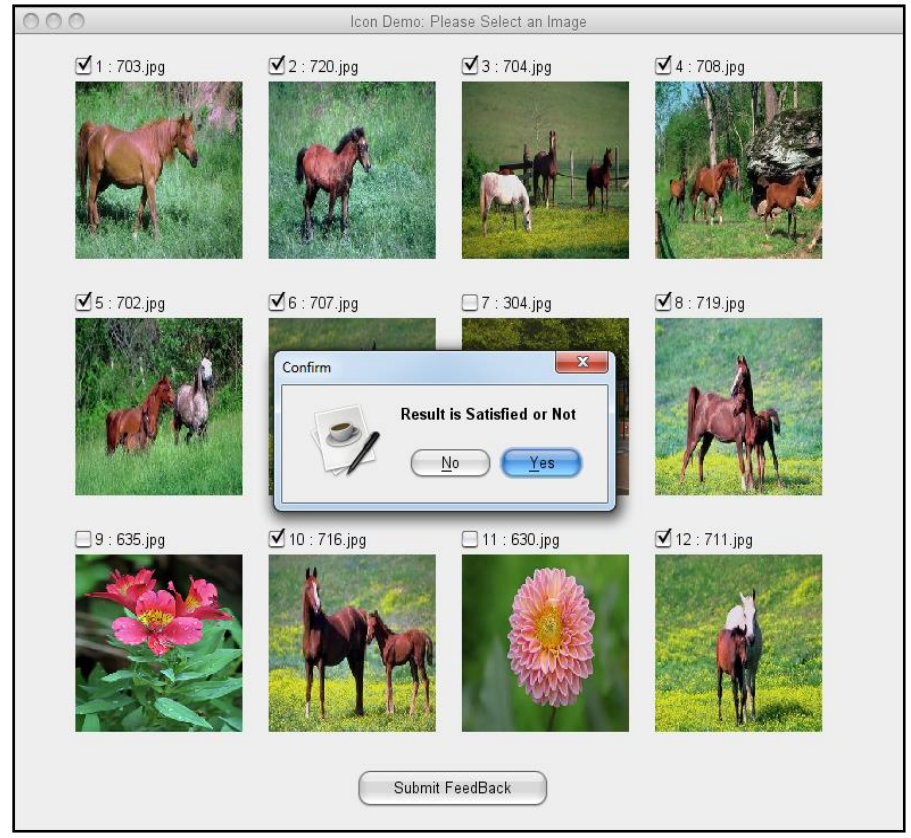

Fig. 10. User satisfaction

\section{NPRF Search:}

In this search new query points are generated and by using NPRF algorithm it produce relevant images by clicking get image button and finally we get relevant images which are relevant to input image. 


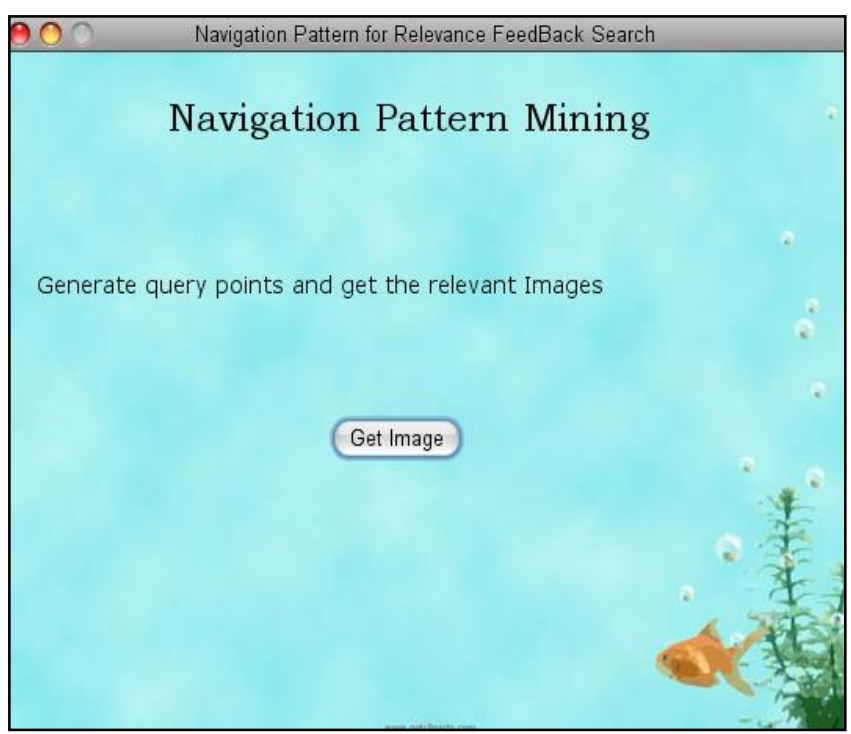

Fig. 11. NPRF search

Result:

Final result is produced which are relevant to query image given by the user.

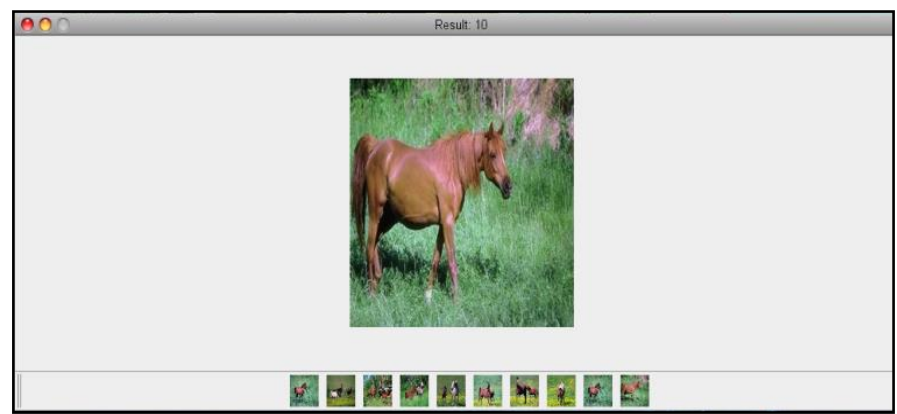

Fig. 12. Final result

\section{Add Image:}

Any image from the user's system is added to the database and one can retrieve image from that database.

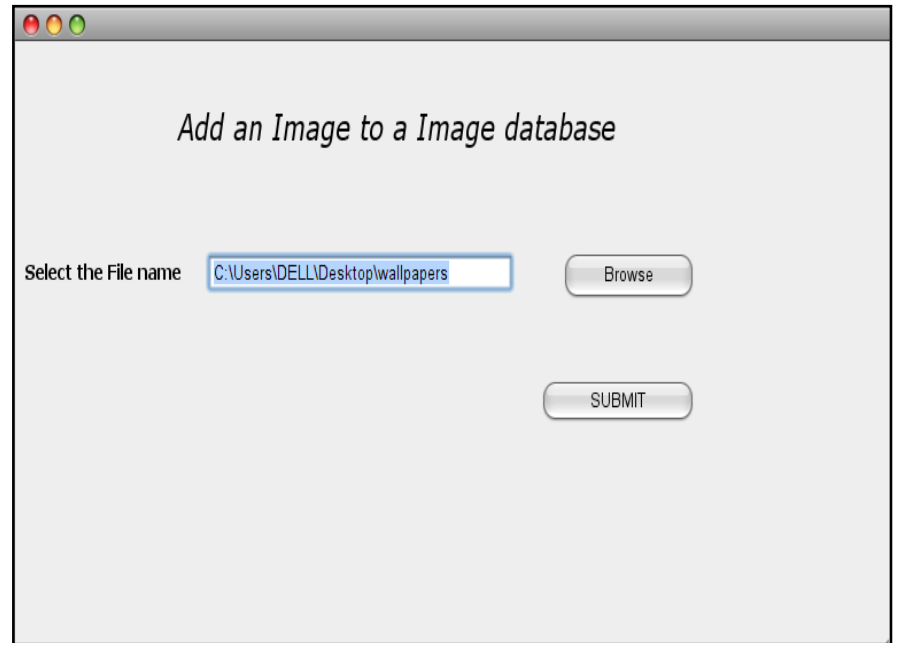

Fig. 13. Add Image

\section{Insert image:}

If image is inserted into database then we get message box i.e. image is inserted successfully then click "ok" button.

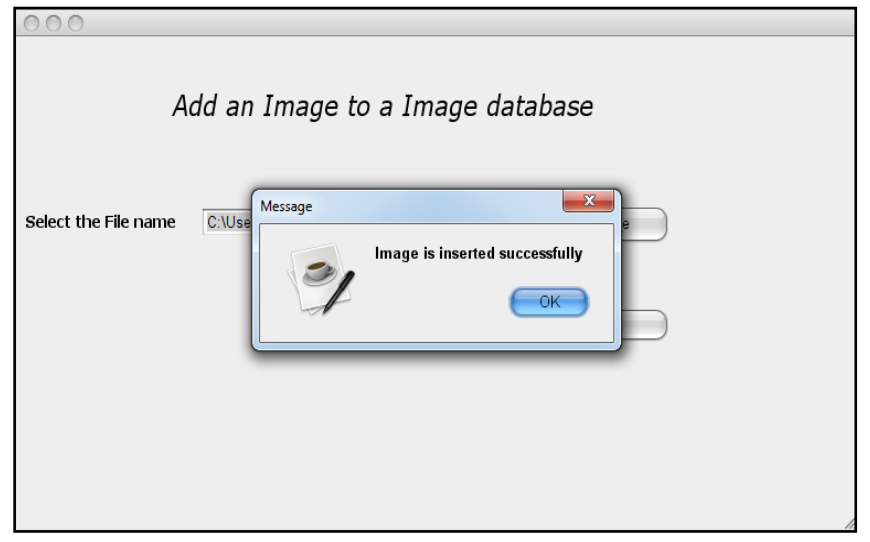

Fig. 14. Insert Image

\section{Retrieving Image:}

Added image is to our database is "null.jpg" image which is not in our database is c:lusers|DELL/Desktoplwallpapers is retrieved for next retrieval.

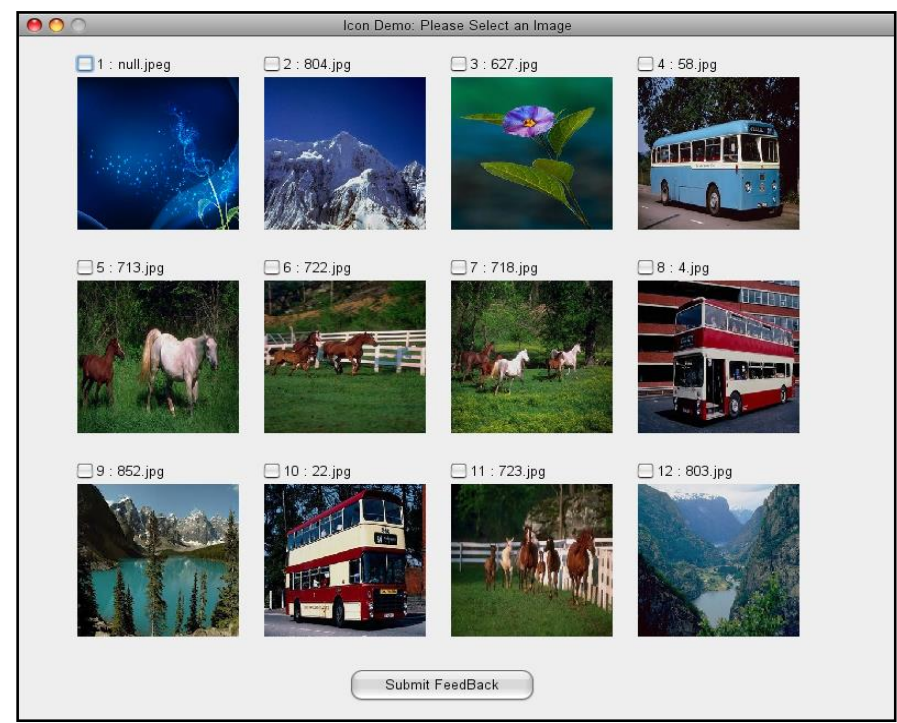

Fig. 15. Retrieving Image

\section{CONCLUSION}

To solve the problem of long series of interaction with the user, the iteration technique included in CBIR combined with $\mathrm{RF}$ formulates into a novel method. So we have proposed a new approach called NPRF- navigation pattern based relevance feedback. The main aim of this approach is to get efficient results coupled with getting high quality of image retrieval with optimal or few feedbacks. Our approach will satisfy the user's intention from a long term search activity or browsing. In NPRF approach, we satisfy the user's intention by merging three query refinement strategies $\mathrm{QR}, \mathrm{QEX}$ and QPM. As a result, problems occurred in existing systems like redundant browsing and visual diversity are solved. The experimental results of the proposed approach are evaluated by using precision, recall measures. 


\section{REFERENCES}

[1] M.D. Flickner, H. Sawhney, W. Niblack, J. Ashley, Q. Huang, B.Dom, M. Gorkani, J. Hafner, D. Lee, D. Steele, and P. Yanker,"Query by Image and Video Content: The QBIC System," Computer, vol. 28, no. 9, pp. 23-32, Sept. 1995.

[2] D. Harman, "Relevance Feedback Revisited," Proc. 15th Ann. Int'1 ACM SIGIR Conf. Research and Development in Information Retrieval, pp. 1-10, 1992.

[3] Y. Ishikawa, R. Subramanya, and C. Faloutsos, "MindReader: Querying Databases through Multiple Examples," Proc. 24th Int'l Conf. Very Large Data Bases (VLDB), pp. 218-227, 1998.

[4] K. Porkaew, K. Chakrabarti, and S. Mehrotra, "Query Refinement for Multimedia Similarity Retrieval in MARS," Proc. ACM Int'l Multimedia Conf. (ACMMM), pp. 235-238, 1999.

[5] A. Pentalnd, R.W. Picard, and S. Sclaroff, "Photobook: Content-Based Manipulation of Image Databases," Int'1 J. Computer Vision (IJCV), vol. 18, no. 3, pp. 233-254, June 1996.

[6] Y. Rui, T. Huang, and S. Mehrotra, "Content-Based Image Retrieval with Relevance Feedback in MARS," Proc. IEEE Int'l Conf. Image Processing, pp. 815-818, Oct. 1997.

[7] J.R. Smith and S.F. Chang, "VisualSEEK: A Fully Automated ContentBased Image Query System,” Proc. ACM Multimedia Conf., Nov. 1996.

[8] G. Salton and C. Buckley, "Improving Retrieval Performance by Relevance Feedback," J. Am. Soc. Information Science, vol. 41, no. 4, pp. 288-297, 1990.

[9] K. Vu, K.A. Hua, and N. Jiang, "Improving Image Retrieval Effectiveness in Query-by-Example Environment," Proc. 2003 ACM Symp. Applied Computing, pp. 774-781, 2003.

[10] L. Wu, C. Faloutsos, K. Sycara, and T.R. Payne, "FALCON: Feedback Adaptive Loop for Content-Based Retrieval," Proc. 26th Int'l Conf. Very Large Data Bases (VLDB), pp. 297-306, 2000.

[11] P.Y. Yin, B. Bhanu, K.C. Chang, and A. Dong, "Integrating Relevance Feedback Techniques for Image Retrieval Using Reinforcement Learning," IEEE Trans. Pattern Analysis and Machine Intelligence, vol. 27, no. 10, pp. 1536-1551, Oct. 2005.
[12] A.Sri Nagesh, Dr.G.P.S.Varma, Dr A Govardhan, "An Improved iterative Watershed and Morphological Transformation Techniques for Segmentation of Microarray Images" , IJCA Special Issue on "Computer Aided Soft Computing Techniques for Imaging and Biomedical Applications" CASCT, Volume 2, 2010.PP 77-87,August 2010.

[13] C.Gonzalez, R.E.Woods, Digital Image Processing, third ed., Prentice Hall, 2007

[14] H. You, E. Chang, and B. Li, "NNEW: Nearest Neighbor Expansion by Weighting in Image Database Retrieval," Proc. IEEE Int'l Conf. Multimedia and Expo, pp. 245-248, Aug. 2001.

[15] X.S. Zhou and T.S. Huang, "Relevance Feedback for Image Retrieval: A Comprehensive Review," Multimedia Systems, vol. 8, no. 6, pp. 536544, Apr. 2003.

[16] D.H. Kim and C.W. Chung, "Qcluster: Relevance Feedback Using Adaptive Clustering for Content-Based Image Retrieval," Proc. ACM SIGMOD, pp. 599-610, 2003.

[17] Petrakis E. and Faloutsos A., "Similarity searching in medical image databases," Journal of IEEE Transaction on Knowledge and Data Engineering, vol. 9, pp435-447, 1997.

[18] A.Sri Nagesh, Dr.G.P.Saradhi Varma, Dr.A.Govardhan \& Dr.B.Raveendra Babu, "An Analysis and Comparison of Quality Index Using Clustering Techniques for Spot Detection in Noisy Microarray Images". International Journal of Image Processing (IJIP), pp 504-511, Volume (5): Issue (4): 2011, CSC Journals, Kuala Lumpur, Malaysia, ISSN: 1985-2304.

[19] Dr. Fuhui Long, Dr. Hongjiang Zhang and Prof. David Dagan Feng, "fundamentals of content-based image retrieval.J. Weszka, C. Dyer, and A. Rosenfeld. A comparative study of texture measures for terrain classification. IEEE Transactions on Systems, Man and Cybernetics, 6(4):269-285, 1976.

[20] Remco C. Veltkamp and Mirela Tanase, "Content Based Image Retrieval Systems: A Survey," International Journal of Engineering Science and Technology, Vol. 20, No. 5, pp. 1-62, 2002.

[21] Armitage, L and Enser, P G B (1997) "Analysis of user need in image archives.” Journal of Information Science, 23(4), 287-299. 